\title{
ESCRT-III is necessary for the integrity of the nuclear envelope in micronuclei but is aberrant at ruptured micronuclear envelopes generating damage
}

\author{
Jessica Willan 1,2, Alexa J. Cleasby ${ }^{1}$, Neftali Flores-Rodriguez ${ }^{3}$, Flavia Stefani ${ }^{4}$, Cinzia Rinaldo ${ }^{5,6}$, Alessandra Pisciottani ${ }^{5}$, \\ Emma Grant ${ }^{1,2}$, Philip Woodman (10), Helen E. Bryant $\mathbb{1}^{2}$ and Barbara Ciani (i)
}

\begin{abstract}
Micronuclei represent the cellular attempt to compartmentalize DNA to maintain genomic integrity threatened by mitotic errors and genotoxic events. Some micronuclei show aberrant nuclear envelopes (NEs) that collapse, generating damaged DNA that can promote complex genome alterations. However, ruptured micronuclei also provide a pool of cytosolic DNA that can stimulate antitumor immunity, revealing the complexity of micronuclear impact on tumor progression. The ESCRT-III (Endosomal Sorting Complex Required for Transport-III) complex ensures NE reseals during late mitosis and is repaired in interphase. Therefore, ESCRT-III activity maybe crucial for maintaining the integrity of other genomic structures enclosed by a NE. ESCRT-III activity at the NE is coordinated by the subunit CHMP7. We show that CHMP7 and ESCRT-III protect against the genomic instability associated with micronuclei formation. Loss of ESCRT-III activity increases the population of micronuclei with ruptured NEs, revealing that its NE repair activity is also necessary to maintain micronuclei integrity. Surprisingly, aberrant accumulation of ESCRT-III are found at the envelope of most acentric collapsed micronuclei, suggesting that ESCRT-III is not recycled efficiently from these structures. Moreover, CHMP7 depletion relieves micronuclei from the aberrant accumulations of ESCRT-III. CHMP7-depleted cells display a reduction in micronuclei containing the DNA damage marker RPA and a sensor of cytosolic DNA. Thus, ESCRT-III activity appears to protect from the consequence of genomic instability in a dichotomous fashion: ESCRT-III membrane repair activity prevents the occurrence of micronuclei with weak envelopes, but the aberrant accumulation of ESCRT-III on a subset of micronuclei appears to exacerbate DNA damage and sustain proinflammatory pathways.
\end{abstract}

Correspondence: Philip Woodman (philip.woodman@manchester.ac.uk) or Helen E. Bryant (h.bryant@sheffield.ac.uk) or Barbara Ciani

(b.ciani@sheffield.ac.uk)

${ }^{1}$ Department of Chemistry, Centre for Membrane Interactions and Dynamics (CMIAD), Krebs Institute, University of Sheffield, Brook Hill, Sheffield S3 7HF, United Kingdom

${ }^{2}$ Academic Unit of Molecular Oncology, Sheffield Institute for Nucleic Acids (SInFoNiA), Department of Oncology and Metabolism, University of Sheffield, Beech Hill Road, Sheffield S10 2RX, United Kingdom

Full list of author information is available at the end of the article.

These authors contributed equally: Jessica Willan, Alexa J. Cleasby, Neftali

Flores-Rodriguez

\section{Introduction}

Micronuclei are cytosolic chromatin structures that are compartmentalized by a nuclear envelope (NE). They are a measure of chromosome instability and thus are hallmarks of cancer cells. Micronuclei originate during mitosis, either because whole chromosomes separate aberrantly, or because DNA damage generates chromosomal fragments that lack centromeres and thus fail to align at the metaphase plate ${ }^{1}$. The resulting structures rebuild their own NE away from the main chromatin mass. Micronuclei persist through multiple cell divisions,

\section{(c) The Author(s) 2019}

(c) (i) Open Access This article is licensed under a Creative Commons Attribution 4.0 International License, which permits use, sharing, adaptation, distribution and reproduction c. in any medium or format, as long as you give appropriate credit to the original author(s) and the source, provide a link to the Creative Commons license, and indicate if changes were made. The images or other third party material in this article are included in the article's Creative Commons license, unless indicated otherwise in a credit line to the material. If material is not included in the article's Creative Commons license and your intended use is not permitted by statutory regulation or exceeds the permitted use, you will need to obtain permission directly from the copyright holder. To view a copy of this license, visit http://creativecommons.org/licenses/by/4.0/. 
but their NE can collapse within the G2 phase of the cell cycle $^{2}$. The cause of such collapse remains unclear, but correlates with a lack of nuclear lamina integrity ${ }^{2}$. Such loss of micronuclear compartmentalization causes cytosolic enzymes to enter the micronucleus, generating further DNA damage and chromosome pulverization ${ }^{3}$. A micronucleus can reincorporate in the primary nucleus, creating the conditions for DNA fragments to rejoin the main genome at random locations (chromothripsis). Therefore, an intact NE around a micronucleus maintains the integrity of its genetic material ${ }^{4-6}$ and thereby protects against chromothripsis.

$\mathrm{NE}$ integrity at the primary nucleus is ensured by ESCRT-III, a universal membrane-remodeling complex. Specifically, ESCRT-III seals nuclear membranes during late mitosis and repairs mechanical rupture of the NE during interphase ${ }^{7-10}$. Core ESCRT-III subunits, including the critical membrane-deforming polymer CHMP4B (Charged Multivesicular body Protein 4B), are recruited by $\mathrm{CHMP7}^{11}$, a specialized ESCRT-III subunit that is targeted to NE gaps by associating with the chromatin binding protein LEM2 ${ }^{12}$. ESCRT-III seals these gaps by supporting reverse-topology membrane scission ${ }^{13}$. ESCRT-III activity at the NE is short-lived, and is regulated by the AAA ATPase, VPS4. VPS4 remodels ESCRTIII to drive membrane scission, and also recycles ESCRTIII subunits back into the cytosol ${ }^{13}$.

Loss of micronuclear compartmentalization exposes DNA to the cytosol, which drives protective immune responses ${ }^{14}$. Cytosolic DNA is recognized as foreign by innate immune pathways involving cyclic GAMP synthase $(\text { cGAS })^{15}$. cGAS binds to specific secondary structures within exposed double and single-stranded DNA and stimulates the production of $2^{\prime}-5^{\prime}$ cyclic GMP-AMP $(\text { cGAMP) })^{16}$.

Here we present how ESCRT-III protects the genome from the instability generated by micronuclei. We show that ESCRT-III and VPS4 support micronuclear envelope membrane integrity, mirroring their role in maintaining the primary $\mathrm{NE}^{17}$. We also show that ESCRT-III activity is aberrant at acentric collapsed micronuclei in unperturbed cancer cells. We interpret this aberrant activity of ESCRTIII as necessary for maintaining a population of ruptured, cGAS-enriched micronuclei. Consistent with such a role, this pool of collapsed micronuclei containing ESCRT-III, cGAS and ssDNA is maintained by an apparent impairment in ESCRT-III recycling and is removed by preventing ESCRT-III recruitment at the NE.

\section{Results}

Depletion of CHMP7 or VPS4 compromise the NE but damage DNA in different fashion

ESCRT-III helps to re-form the NE during cell division and repairs the NE during interphase ${ }^{7,9,10,18}$. To further understand the impact of ESCRT-III on NE integrity in micronuclei, we first performed a detailed analysis of the phenotypes observed at interphase nuclei when ESCRTIII activity is impaired. We depleted CHMP7 (Supplemental Fig. 1A, B), which initiates NE-associated ESCRTIII assembly ${ }^{11}$, and VPS4 (Supplemental Fig. 1C and see Methods), which regulates ESCRT-III-mediated membrane-remodeling activity ${ }^{17}$.

Depletion of CHMP7 or VPS4 increased the frequency of multinucleated cells (Supplemental Fig. 1D) and increased the proportion of deformed interphase nuclei (Supplemental Fig. 1E), confirming their functional knockdown ${ }^{19}$. Focusing on the role of ESCRT-III in NE sealing, we observed defects in NE structure within interphase nuclei. Consistent with CHMP7-initiating NE sealing, depletion of this protein generated gross defects in the nuclear lamina, including honeycomb-like and herniated breaks (Fig. 1a, b). VPS4 depletion did not generate such severe abnormalities (Fig. 1b), but resulted in discontinuities in lamin B staining. These discontinuities were enriched for CHMP7, as well as lamin A/C and LAP2 (Fig. 1c-e), indicating enrichment in membrane with CHMP7 accumulations. Large CHMP7 accumulations were seen in $>80 \%$ of VPS4-depleted cells (Fig. 1f, g), but in only $<1 \%$ of interphase untreated or control-treated cells. CHMP7 accumulations in VPS4-depleted cells also contained CHMP4B (Fig. 1f, h), whereas CHMP4B was absent when cells were co-depleted for VPS4 and CHMP7 (Fig. 1h), in agreement with CHMP7 coordinating the recruitment of CHMP4B at the NE.

Consistent with their function at the NE, CHMP7 or VPS4 depletion caused a loss of nuclear compartmentalization, as revealed by the presence in the cytoplasm of ProMyelocytic Leukemia (PML) bodies ${ }^{20}$ (Supplemental Fig. 2A, B). In addition, h-TERT human fibroblasts depleted of CHMP7 displayed an increased cytosolic distribution of a general nuclear import marker (GFPNLS, Supplemental Fig. 2A, C and Supplemental Fig. 1F). CHMP7 and VPS4 depletion generated substantial DNA damage as assessed by staining with $\gamma \mathrm{H} 2 \mathrm{AX}$, a marker for double-strand breaks ${ }^{21,22}$. Upon CHMP7 depletion (Supplemental Fig. 2D), $\gamma \mathrm{H} 2 \mathrm{AX}$ stained abundant foci throughout the nucleus, which were large compared with the rare $\gamma \mathrm{H} 2 \mathrm{AX}$ foci seen in control cells (Supplemental Fig. 2E, F). Abundant $\gamma \mathrm{H} 2 \mathrm{AX}$ foci were also seen in VPS4-depleted cells (Supplemental Fig. 2E), nevertheless these were generally smaller than those seen in CHMP7-depleted cells (Supplemental Fig. 2F), and confined towards the nuclear periphery surrounding large CHMP7 accumulations (Supplemental Fig. 2G top panels). CHMP7 foci in VPS4-depleted cells also labeled for the DNA repair markers Rad51 and RPA (Supplemental Fig. 2G, H), indicating the presence of singlestranded DNA (ssDNA) ${ }^{23}$. 
A
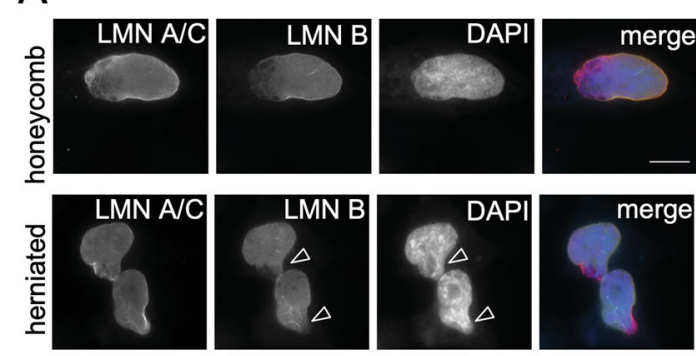

C

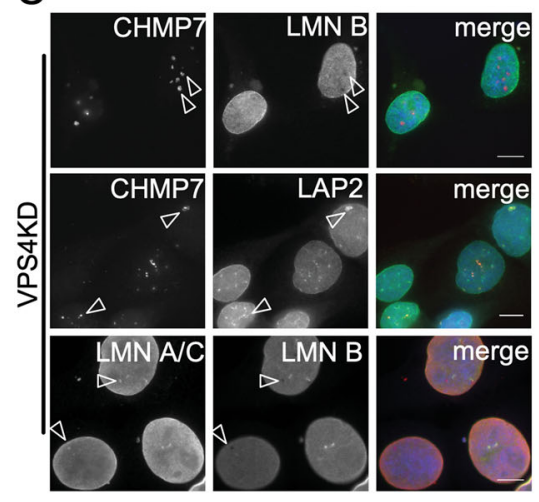

$\mathrm{D}$

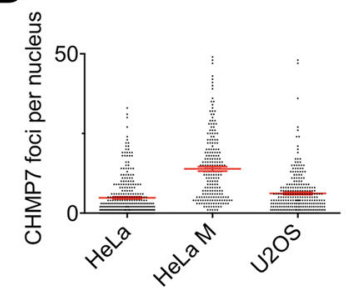

$\mathrm{E}$
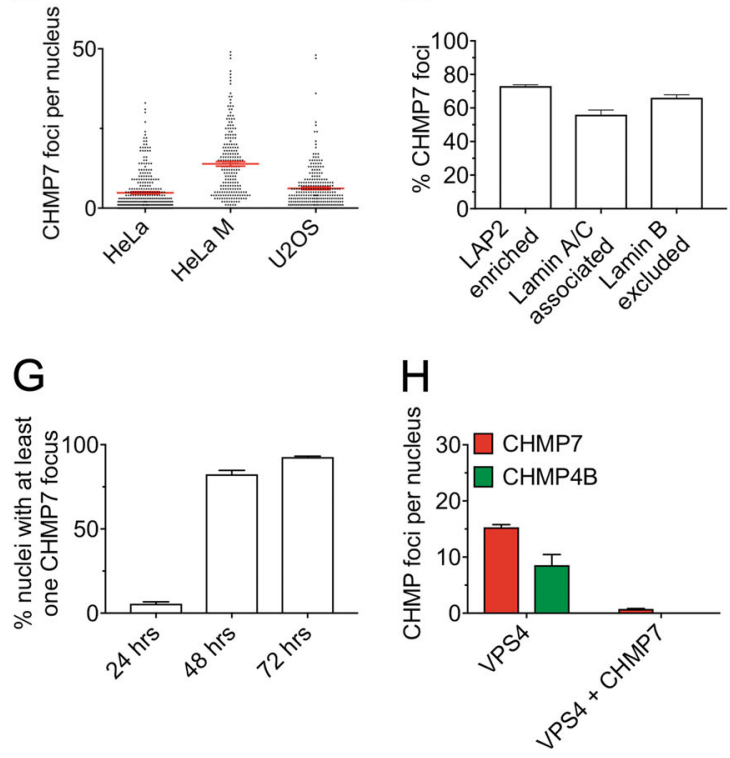

B
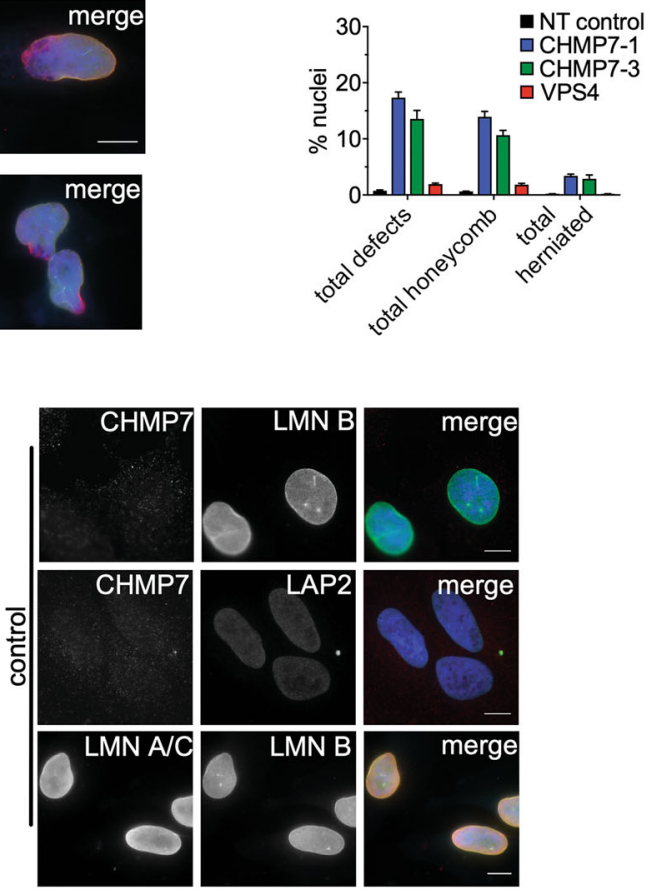

$\mathrm{F}$

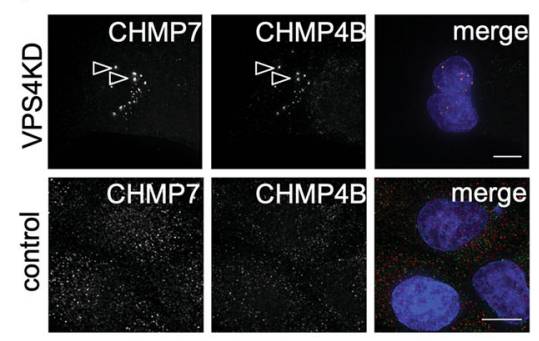

Fig. 1 CHMP7 and VPS4 depletion cause general nuclear envelope disorganization. a Examples of the honeycomb and herniated Lamin A and B phenotypes in HeLa cells treated with CHMP7 sRNAi for $48 \mathrm{~h}$. Arrowheads indicate chromatin protruding out the nucleus in herniated cells. $\mathbf{b}$ Quantification of Lamin A and B defects in Hela cells treated with the indicated sRNAi (minimum of 300 cells scored per condition). $\mathbf{c}$ Examples of CHMP7 foci in HeLa cells treated with VPS4 and NT-control sRNAi for $48 \mathrm{~h}$ and associated with LAP2 or Lamin A foci (LAP2 and Lamin A enriched, arrowheads) or associated with Lamin B holes (HeLa M). d Average CHMP7 foci number per nucleus in HeLa ( $n=305)$, HeLa M ( $n=222)$, and U2OS $(n=257)$ cells treated with VPS4 sRNAi for $48 \mathrm{~h}$. e Quantification of CHMP7 foci in relation to LAP2, Lamin A/C, or Lamin B, in HeLa cells treated with VPS4 sRNAi for $48 \mathrm{~h}$ (minimum of 400 foci for each condition). $\mathbf{f}$ Deconvolved widefield image of CHMP4B co-localization with CHMP7 at nuclear envelope foci in HeLa cells transfected with VPS4 sRNAi for $48 \mathrm{~h}$ (top panel) and CHMP7/CHMP4B distribution in control cells (bottom panel). $\mathbf{g}$ Percentage of HeLa cells containing at least one large accumulation of CHMP7 (focus) following VPS4 sRNAi treatment at 24, 48, and 72 h post transfection (minimum of 200 nuclei scored for each condition). $\mathbf{h}$ Number of nuclear CHMP foci accumulating in HeLa M cells under the indicated sRNAi treatments. (minimum of 100 nuclei scored for each condition). Averages and SEM are shown $(N=3)$. Scale bars represent $10 \mu \mathrm{m}$ 
Overall, these data reflect the difference between coordination of recruitment (CHMP7) and dynamics of ESCRT-III assembly (VPS4) at the $\mathrm{NE}^{7,9,10}$. CHMP7depleted cells are defective in NE assembly and show widespread lamina disorganization, rupture and DNA damage. In contrast, VPS4 depletion induces aberrant ESCRT-III accumulations that associate with localized NE membrane enrichment and ssDNA.

\section{Loss of ESCRT-III activity increases micronuclei with compromised envelope membranes in interphase}

Cells displaying chromosomal instability are characterized by micronuclei, which are generated when lagging chromosomes or chromosome fragments are enveloped by NE membranes. Lagging DNA arises directly from kinetochore-microtubule attachment errors during mitosis (aneugenic mechanisms), as well as an indirect consequence of unrepaired DNA damage during previous cell cycles that generated chromosome fragments (clastogenic mechanisms) $)^{21,24,25}$. Given the roles of ESCRT-III at the nucleus, mitosis and cell division ${ }^{10,18,26,27}$, we assessed the impact of impairing ESCRT-III function on the incidence of micronuclei with ruptured envelopes.

Depletion of CHMP7 and/or VPS4 resulted in at least approximately twofold increase in the population of cells containing micronuclei (Fig. 2a, b) and a modest increase in the number of micronuclei per micronucleated cell (Fig. 2c). Micronuclei arising from clastogenic events ${ }^{28,29}$ are acentric, whereas micronuclei arising from aneugenic events contain whole chromosomes and therefore contain centromeres. Approximately $50 \%$ of micronuclei in control cells contained centromeres, as assessed by staining for the centromeric marker CREST. This proportion changed only slightly upon depletion of either CHMP7 or VPS4, albeit with opposite tendencies (Fig. 2d). Hence, the increase in micronuclei resulting from ESCRT-III depletion most likely arises via a combination of aneugenic and clastogenic mechanisms.

We then examined lamin B staining to assess the impact of ESCRT-III disruption on the NE of micronuclei. Depletion of VPS4 or CHMP7 increased the proportion of micronuclei in which the lamina was absent or discontinuous (Fig. 2e; Supplemental Fig. 3A), recapitulating the phenotype observed at the primary nucleus. Micronuclei lacking nuclear pore complexes (NPCs) may be unable to import lamins, and indeed the density of NPCs at micronuclei, assessed by mab414 staining (Supplemental Fig. 3B, C), was reduced upon VPS4 or CHMP7 depletion (Fig. 2f). Disruption of NE architecture can lead to invasion of the endoplasmic reticulum (ER) into the micronucleus core ${ }^{2}$. Depletion of CHMP7 or VPS4 increased ER invasion, as assessed by intense labeling of micronuclei with the lumenal ER marker Protein Disulfide Isomerase (PDI) (Figs. $2 \mathrm{~g}$ and $5 \mathrm{a}, \mathrm{c}$ ). Moreover, fewer micronuclei in CHMP7 or VPS4-depleted cells labeled for the soluble nuclear enzyme PARP1 (Fig. 2h) or for GFPNLS (Fig. 2i, j), indicating compromised compartmentalization. In summary, loss of ESCRT-III function promotes formation micronuclei that largely show impaired NE integrity.

\section{ESCRT-III accumulates within a subset of micronuclei}

Interestingly, we observed a significant enrichment of ESCRT-III proteins at the NE of micronuclei in unperturbed cells. Therefore, we examined the morphology of the structures that displayed localization of endogenous ESCRT-III. ESCRT-III residency at the reforming NE of the primary nucleus is dynamic and short-lived ${ }^{10}$, such that large ESCRT-III accumulations are very rarely detected at the interphase nucleus at steady state but are observed in $>80 \%$ cells when VPS4 is depleted (Fig. 1h). To our surprise, however, we found that at micronuclei the situation is strikingly different. Specifically, intense CHMP7 labeling (CHMP7 + ve) was found on 20-25\% of micronuclei in non-treated or control-treated cells (Fig. $3 \mathrm{a}-\mathrm{c}$ ), revealing an accumulation never seen at the primary nucleus and pointing towards a potential dysfunction of ESCRT-III at micronuclei. CHMP7 labeling of micronuclei was observed at a similar frequency across several cancer cell lines (Fig. 3b). CHMP7-labeled micronuclei also stained strongly for CHMP4B (Fig. 3a, d). Whereas CHMP7 labeling was confined to peripheral puncta upon VPS4KD (Supplemental Fig. 2G), Stimulated emission depletion microscopy (STED) revealed that in most (32/35) micronuclei that labeled for ESCRT-III, the staining localized deep within the micronucleus core (Fig. 3d, e). When VPS4 was depleted, the population of CHMP7-enriched micronuclei rose to 60\% (Fig. 3d). Thus, an additional pool of ESCRT-III associates dynamically ESCRT-III with micronuclei, consistent with ESCRT-III mediated repair (Fig. 2). Recruitment of CHMP4B to micronuclei, both in the presence and absence of VPS4, depended on CHMP7 (Fig. 3c). Hence, both dynamic and persistent pools of ESCRT-III at micronuclei assemble in similar order to that observed at the primary nucleus.

\section{ESCRT-III enriched micronuclei lack NE integrity and nuclear compartmentalization}

Overall, these data suggest that ESCRT-III associates with micronuclei dynamically, presumably as part of a repair process, but also point towards a dramatic retention of ESCRT-III under normal conditions at a subset of micronuclei. The deep penetration into micronuclei of ESCRT-III accumulations potentially points to gross structural defects in these structures. Indeed, whilst most CHMP7-negative micronuclei had an intact (i.e., "continuous") lamina, the lamina was either absent from or discontinuous in virtually all CHMP7-positive micronuclei 
A

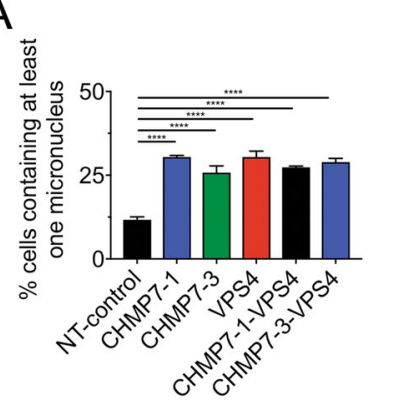

D

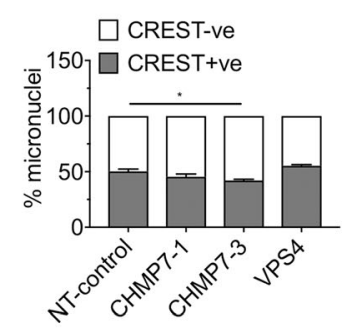

G
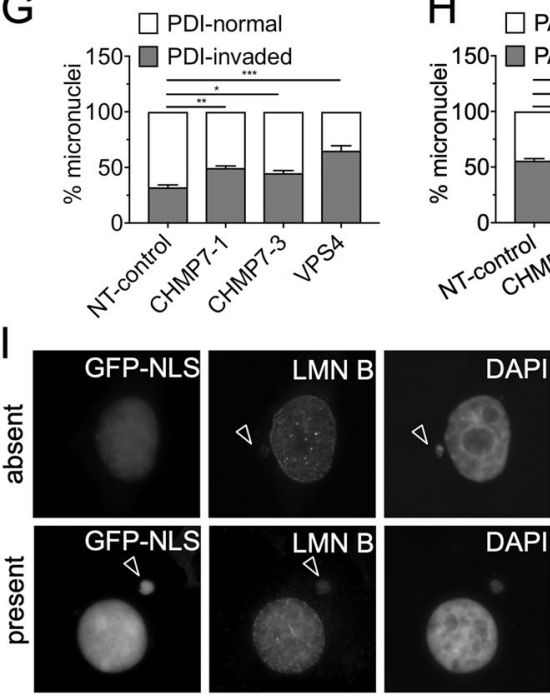

DAPI
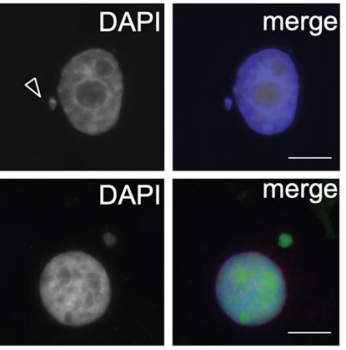

$\mathrm{H}$

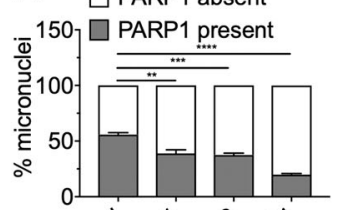

NT.
C

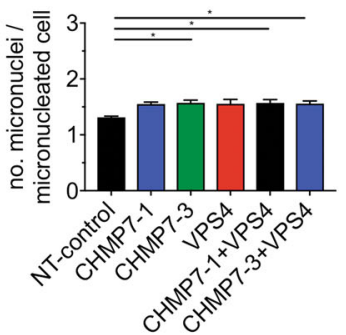

$\mathrm{F}$

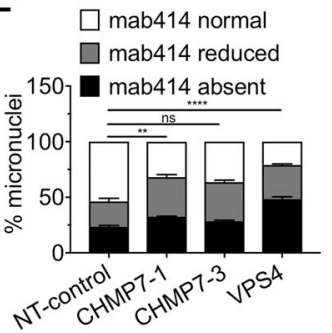

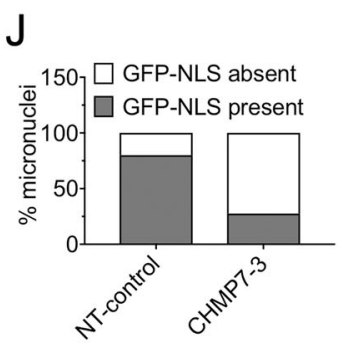

Fig. 2 Impairment of ESCRT-III function affects micronuclei integrity. a HeLa cells were transfected with the indicated sRNAi for $48 \mathrm{~h}$ and the percentages of cells with at least one micronucleus were quantified for each treatment (minimum 250 cells per treatment, per repeat). Results were analyzed using a one-way ANOVA with Dunnett's post hoc test. b HeLa cells were transfected with the indicated sRNAi for either 24, 48, or $72 \mathrm{~h}$. The number of micronucleated cells were quantified for each one of the treatments (minimum 200 cells were scored for each treatment, per repeat). Results were analyzed using a two-way ANOVA with Dunnett's post hoc test. c The average number of micronuclei observed per micronucleated cell were quantified (minimum 150 cells per treatment, per repeat). Results were analyzed using a one-way ANOVA with Dunnett's post hoc test. $\mathbf{d}$ Micronuclei in HeLa cells transfected for $48 \mathrm{~h}$ with the indicated sRNAi and scored for presence of CREST (minimum 200 micronuclei per treatment, per repeat). Results were analyzed using a one-way ANOVA with Dunnett's post hoc test. e HeLa cells were transfected with the indicated sRNAi for $48 \mathrm{~h}$ and scored for the status of Lamin B, as defined in supplementary Figure 2A (minimum 100 micronuclei per treatment, per repeat). Results were analyzed using a one-way ANOVA with Dunnett's post hoc test. Statistics shown for the "absent" category. $\mathbf{f}$ HeLa cells were transfected with the indicated sRNAi for $48 \mathrm{~h}$ and scored for the status of mab414, as defined in supplementary Figure 2B (minimum 100 micronuclei per treatment, per repeat). Results were analyzed using a one-way ANOVA with Dunnett's post hoc test. Statistics shown for the 'absent' category. $\mathbf{g}$ Micronuclei in HeLa cells transfected for $48 \mathrm{~h}$ with the indicated sRNAi and scored for presence of PDI within micronuclear chromatin (PDI-invaded; minimum 100 micronuclei per treatment, per repeat). Results were analyzed using a one-way ANOVA with Dunnett's post hoc test. $\mathbf{h}$ Micronuclei in HeLa cells transfected for $48 \mathrm{~h}$ with the indicated sRNAi and scored for presence of PARP1 (minimum 100 micronuclei per treatment, per repeat). Results were analyzed using a one-way ANOVA with Dunnett's post hoc test. Averages and SEM are shown ( $N=3$, unless stated otherwise). i HeLa cells treated with control or CHMP7 sRNAi, then transfected with GFP-NLS post-seeding and imaged $48 \mathrm{~h}$ post sRNAi depletion. Examples of micronuclei with GFP-NLS absent (top panel) or present (bottom panel) (arrowheads). Scale bar $10 \mu \mathrm{m}$. j Quantification of GFP-NLS retention in micronuclei treated as in $\mathbf{g}$ as judged visually by the loss of nuclear signal ( 25 micronuclei scored per treatment, per repeat). Averages of $N=2$ biological repeats are shown 

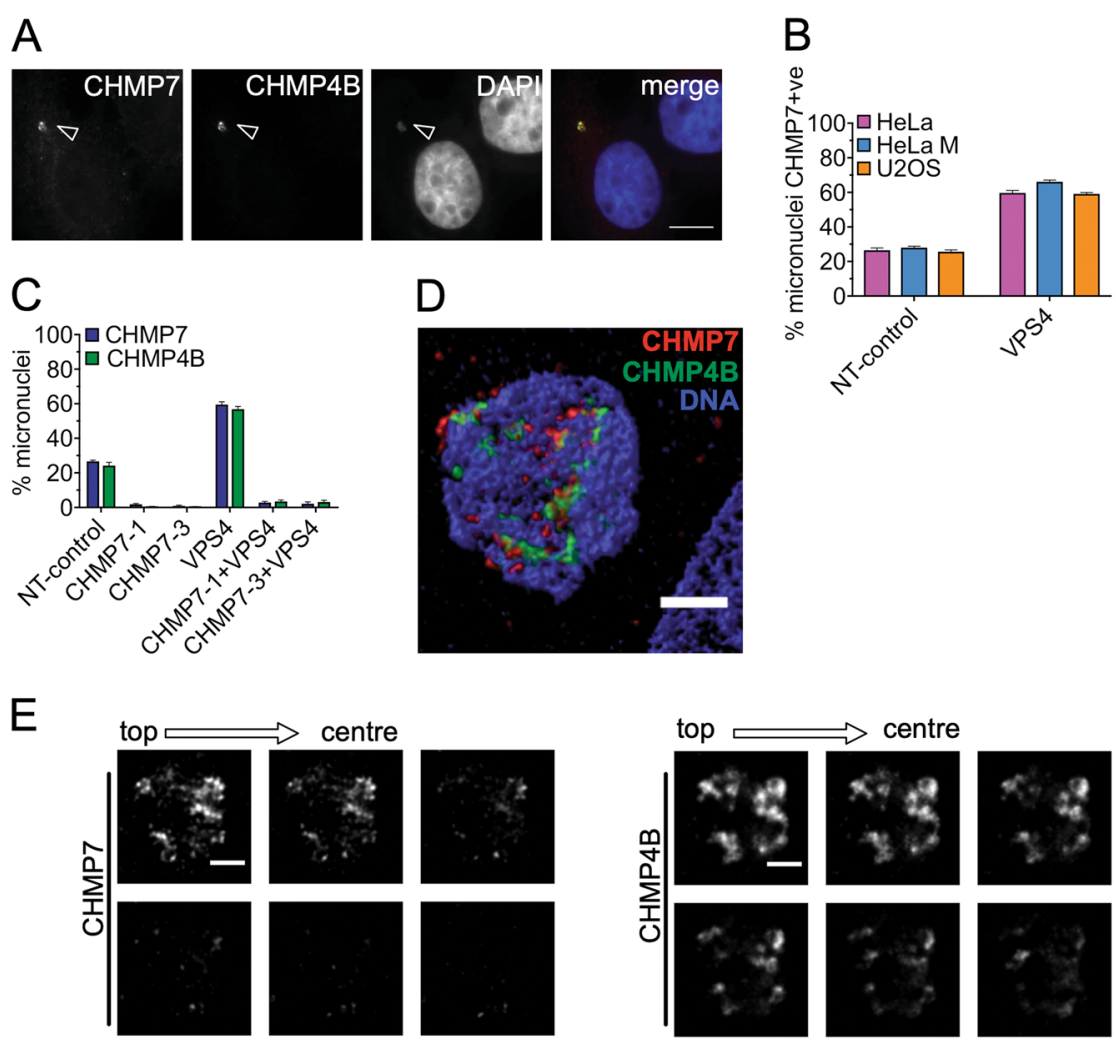

Fig. 3 ESCRT-III accumulates on micronuclei and is recruited by CHMP7. a Untreated HeLa cells showing accumulations of CHMP7 and CHMP4B at a micronucleus (arrowhead). Scale bars $10 \mu \mathrm{m}$. b U2OS, HeLa, and HeLa M cell lines were transfected with non-targeting or VPS4A and B sRNAi for $48 \mathrm{~h}$, and stained for CHMP7. Micronuclei were scored for presence or absence of CHMP7 (minimum of 400 micronuclei scored per treatment). $\mathbf{c}$ Percentage of micronuclei in HeLa cells transfected with the indicated sRNAi for $48 \mathrm{~h}$ displaying enriched CHMP7 or CHMP4 (minimum 200 micronuclei scored per treatment). $\mathbf{d}$ Confocal microscopy in STED mode of a micronucleus in a control HeLa cell containing CHMP7 and CHMP4B accumulations. Scale bars represent $1 \mu \mathrm{m}$. e Confocal microscopy in STED mode of the micronucleus in $\mathbf{d}$ showing z-slicing across $335 \mathrm{~nm}$ starting from the top going towards the inside of the micronucleus. Scale bar $1 \mu \mathrm{m}$. Averages and SEM are shown $(N=3)$

(Fig. 4a, b and Supplementary Figure 3A). As expected, when a discontinuous Lamin B was present, CHMP7 occupied several gaps in Lamin B staining (Fig. 4a).

We then quantified CHMP7-positive micronuclei according to the density of NPCs. Most CHMP7-positive micronuclei did not contain, or had reduced density, of NPCs compared with CHMP7-negative micronuclei (Fig. $4 \mathrm{c}$, d and Supplemental Fig. 3B). CHMP7-positive micronuclei also rarely contained PARP1, in contrast to CHMP7negative micronuclei (Fig. 4e, f), highlighting a defect in compartmentalization. Loss of NE integrity was not owing simply to an absence of membrane, as all CHMP7-positive micronuclei labeled for the NE marker LAP2, and indeed labeled more strongly for this marker than CHMP7negative micronuclei or parent nuclei (Fig. $4 \mathrm{~g}, \mathrm{~h}$ ).

This finding prompted us to investigate if these micronuclei are "collapsed" and hence their chromatin core is invaded by NE/ER membrane ${ }^{2}$. Such micronuclear envelope collapse is induced by nuclear lamina defects, and this weakness is associated with catastrophic DNA damage, genomic instability and inflammation ${ }^{3,14,30,31}$. Indeed, ER membrane was enriched within the core region of nearly all CHMP7-positive micronuclei in control and VPS4-depleted cells, with the PDI signal much more intense than over the rest of the cell (Fig. $5 \mathrm{a}-\mathrm{c}$ ). In contrast, only $~ 10 \%$ of CHMP7-negative micronuclei exhibited a strong PDI signal intensity (Fig. 5a, b). In summary, CHMP7 accumulates on micronuclei that have a disrupted $\mathrm{NE}$, and which are infiltrated with ER membrane.

Taken together, these data show that ESCRT-III accumulates selectively on micronuclei that lack NE integrity and recapitulate the phenotype occurring at the primary nucleus when VPS4 is depleted. Thus, ESCRT-III activity is impaired at a subset of micronuclei in interphase cells.

\section{ESCRT-III accumulates preferentially to acentric micronuclei containing fragmented DNA}

ESCRT-III accumulates on a subset of micronuclei. To determine whether these are derived primarily from aneugenic or clastogenic events, we quantified the 
A
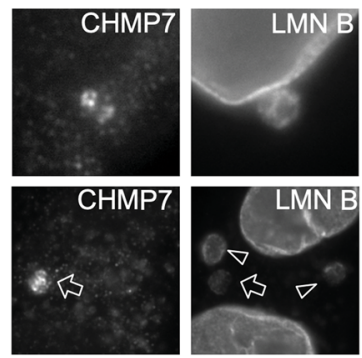

C
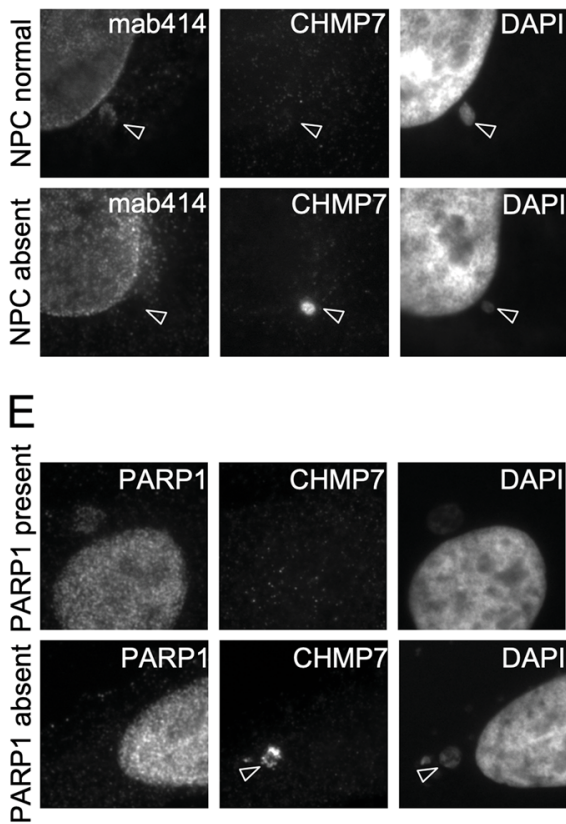

G
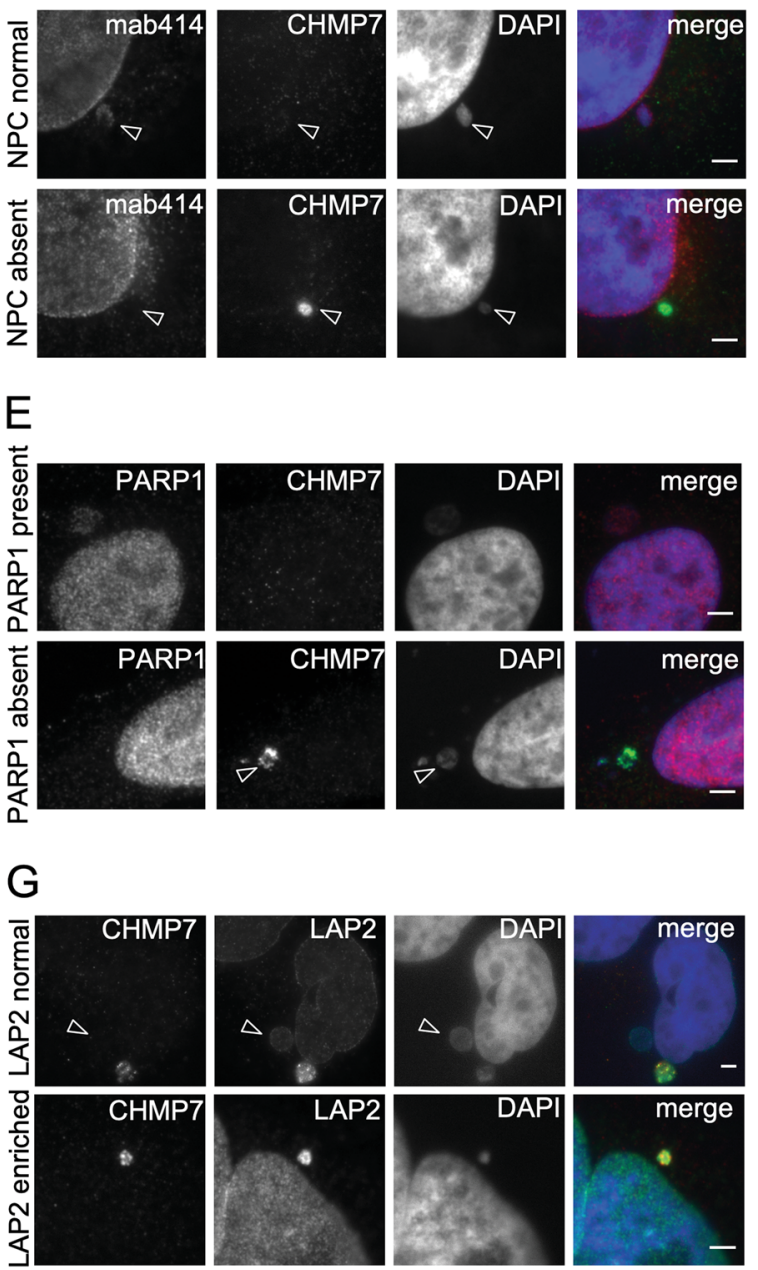

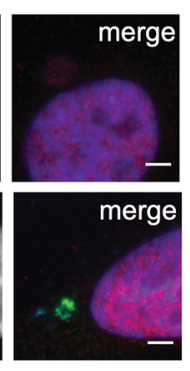

B

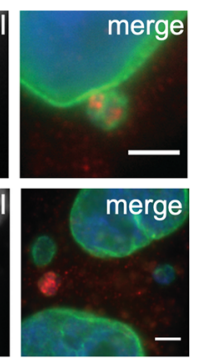

D

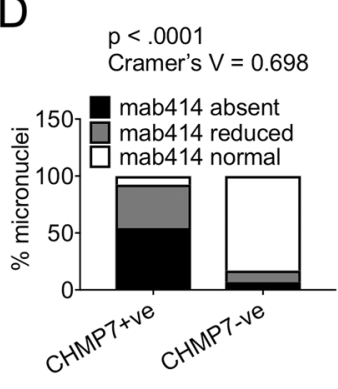

F

$p<.0001$

Cramer's $V=0.660$

$\overline{\bar{\omega}}^{150} \square$ PARP1 absent

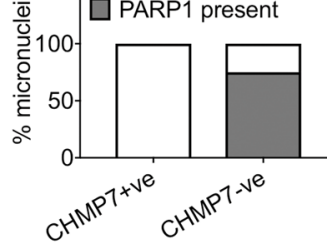

$\mathrm{H}$

$p<.0001$

Cramer's $V=0.842$

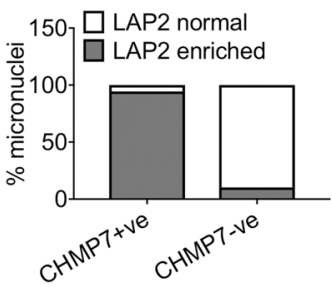

Fig. 4 Micronuclei with CHMP7 accumulations lack nuclear lamina integrity, nuclear pore complexes, and soluble nuclear proteins but are enriched in LAP2. a Immunofluorescence image of a micronucleus where CHMP7 localizes to regions with gaps in Lamin B in control cells (top panel). Examples of CHMP7-positive (arrows) and negative (arrowheads) in control HeLa cells (bottom panel). Scale bar $3 \mu \mathrm{m}$. b Distribution of the status of CHMP7 and Lamin B in micronuclei (minimum 100 micronuclei scored per repeat). c Examples of CHMP7-positive and negative micronuclei with NPC staining in control HeLa cells. $\mathbf{d}$ Micronuclei in HeLa cells were scored for the status of CHMP7 and the status of NPC (mab414) within the same micronucleus (minimum 160 micronuclei scored per repeat). e Examples of CHMP7-positive and negative micronuclei showing absence or presence of PARP1 staining. Scale bar $3 \mu \mathrm{m}$. $\mathbf{f}$ Quantification of the presence or absence of PARP1 and CHMP7 staining within the same micronucleus (minimum 200 micronuclei scored per repeat). $\mathbf{g}$ Examples of CHMP7-positive and negative (arrowhead) micronuclei with LAP2 staining. Scale bar $3 \mu \mathrm{m}$. $\mathbf{h}$ Quantification of the presence or absence of LAP2 and CHMP7 staining within the same micronucleus (300 micronuclei per repeat). Data were analyzed using Fishers' exact test on pooled raw counts distributions $(N=3)$ 
A
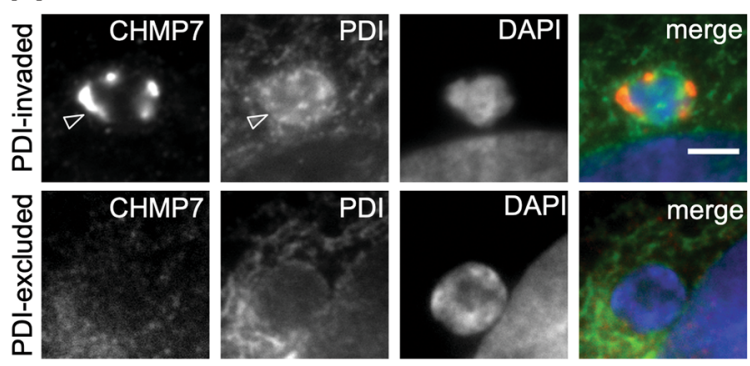

C
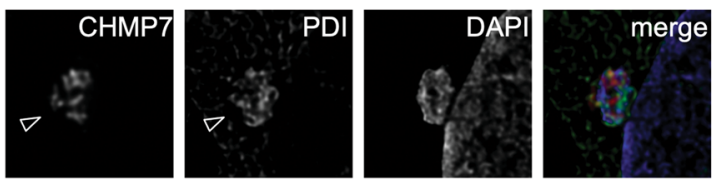

B

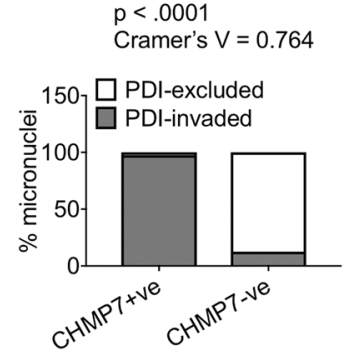

Fig. 5 Micronuclei with ESCRT-III accumulations display ER membrane invasion. a Examples of CHMP7-positive and negative micronuclei with PDI staining. PDI-excluded micronuclei have endoplasmic reticulum (ER) membrane surrounding their boundaries but not in the micronuclear interior. PDI-invaded micronuclei show ER membrane inside the micronuclear boundary, indicating nuclear envelope collapse (arrowheads). $\mathbf{b}$ Distribution of the status of CHMP7 and the presence or absence of PDI invasion within the same micronucleus (minimum 100 micronuclei scored per repeat). Data were analyzed using Fishers' exact test on pooled raw counts distributions ( $N=3$ ). c Example image of a micronucleus in a Hela cell after treatment with VPS4 sRNAi for $48 \mathrm{~h}$. Accumulation of PDI and CHMP7 within the micronucleus and the irregular shape display membrane infiltration (arrowhead). Scale bars $3 \mu \mathrm{m}$

proportion of ESCRT-III micronuclei containing whole or partial chromosomes, using CREST staining ${ }^{28}$. CHMP7labeled micronuclei mostly lacked CREST staining (Fig. 6a, b). In contrast, most CHMP7-negative micronuclei labeled for CREST (Fig. 6a, b). CHMP7-positive micronuclei were also much smaller than CHMP7-negative micronuclei ( $1.5 \mu \mathrm{m}$ in diameter on average compared with $\sim 2.2 \mu \mathrm{m}$, representing a $\sim 50 \%$ smaller volume) (Fig. 6c), consistent with them containing chromosome fragments.

UV-C irradiation is a clastogenic agent, generating single-stranded DNA lesions that can lead via replication stress to double-strand breaks ${ }^{32,33}$ and subsequent aberrant mitotic partitioning of chromosome fragments into micronuclei, which accumulate ssDNA-binding proteins. Therefore, to confirm that ESCRT-III accumulates preferentially on micronuclei arising from DNA damage, we used recovery from UV-C irradiation to selectively increase this population of micronuclei. HeLa cells were irradiated with a moderate dose of $\mathrm{UV}-\mathrm{C}$, and the number and phenotype (i.e., labeling for CHMP7 and/or the double-strand break marker $\gamma \mathrm{H} 2 \mathrm{AX}$ ) of micronuclei were analyzed over $72 \mathrm{~h}$ recovery. The number of micronuclei per cell increased after $24 \mathrm{~h}$ and rose $\sim 4$-fold within $48 \mathrm{~h}$ (Fig. 6d, e), a timing indicative of the need for cells to go through mitosis in order to form micronuclei ${ }^{34}$. Aligned with this increase, the proportion of micronuclei that were CHMP7-positive also rose significantly (Fig. 6f). As expected, labeling of micronuclei for $\gamma \mathrm{H} 2 \mathrm{AX}$ also increased, though here the pattern was more complex.
The population of $\gamma \mathrm{H} 2 \mathrm{AX}$-positive micronuclei first increased modestly around $1 \mathrm{~h}$ post treatment, highlighting a minor population of pre-existing micronuclei that are directly subject to DNA damage (Fig. 6g). A second, larger increase in the proportion of micronuclei labeled for $\gamma \mathrm{H} 2 \mathrm{AX}$, and for both CHMP7 and $\gamma \mathrm{H} 2 \mathrm{AX}$, was apparent over 24-48 h (Fig. 6g, h).

In summary, acentric micronuclei are preferentially enriched by ESCRT-III; consistent with this, clastogenic agents such as $\mathrm{UV}^{21,22,30,35,36}$ increase the population of micronuclei that contain ESCRT-accumulations.

\section{CHMP7 is important for generating ssDNA and retaining cGAS to a pool of micronuclei}

Collectively, our data point to a pool of ESCRT-III that preferentially accumulates on micronuclei lacking NE integrity and compartmentalization. However, ESCRT-III is not the primary cause of these defects in micronuclear organization, as they are more frequent when ESCRT-III function is lacking. As damage to micronuclei can generate a source of immunostimulatory $\mathrm{DNA}^{14}$, we next examined the role of ESCRT-III in this downstream process. Micronuclei enriched for CHMP4B preferentially stained strongly for replication protein A (RPA), a protein that binds directly to ssDNA (Fig. 7a, b). Similarly, cGAS, is known to enter ruptured micronuclei to bind exposed DNA and indeed was concentrated in nearly all those accumulating CHMP7 (Fig. 7c, d). Significantly, CHMP7 depletion reduced the proportion of micronuclei that were enriched for RPA (Fig. 7e, 

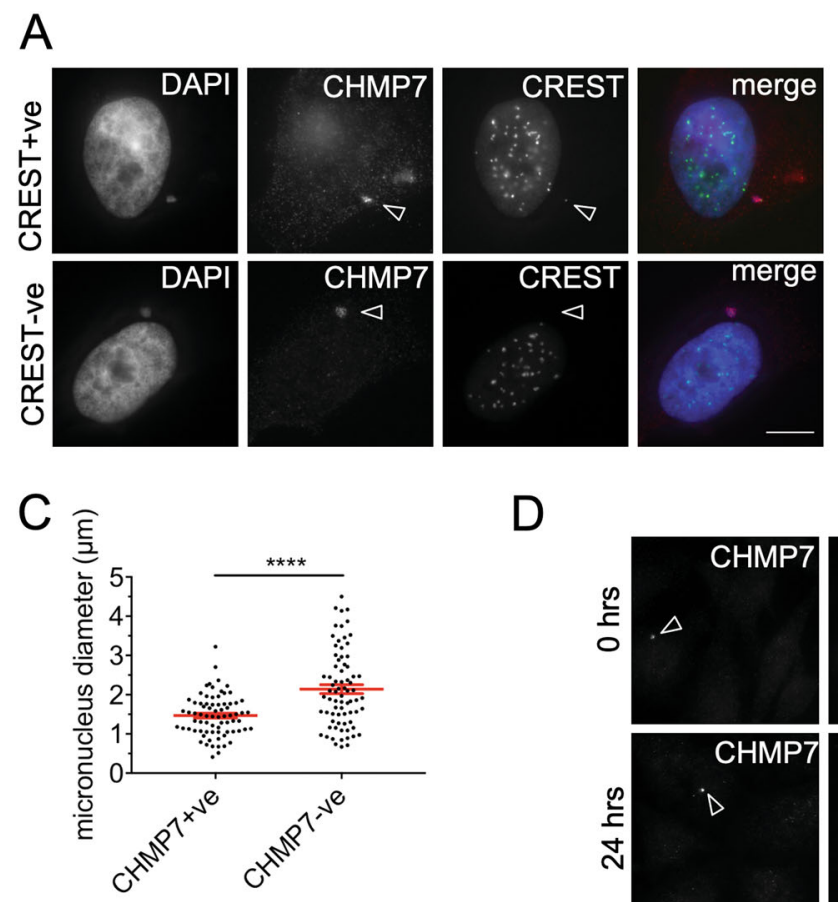

B

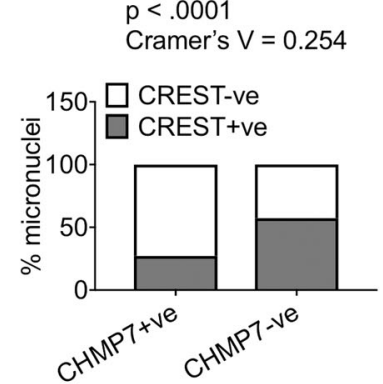

E
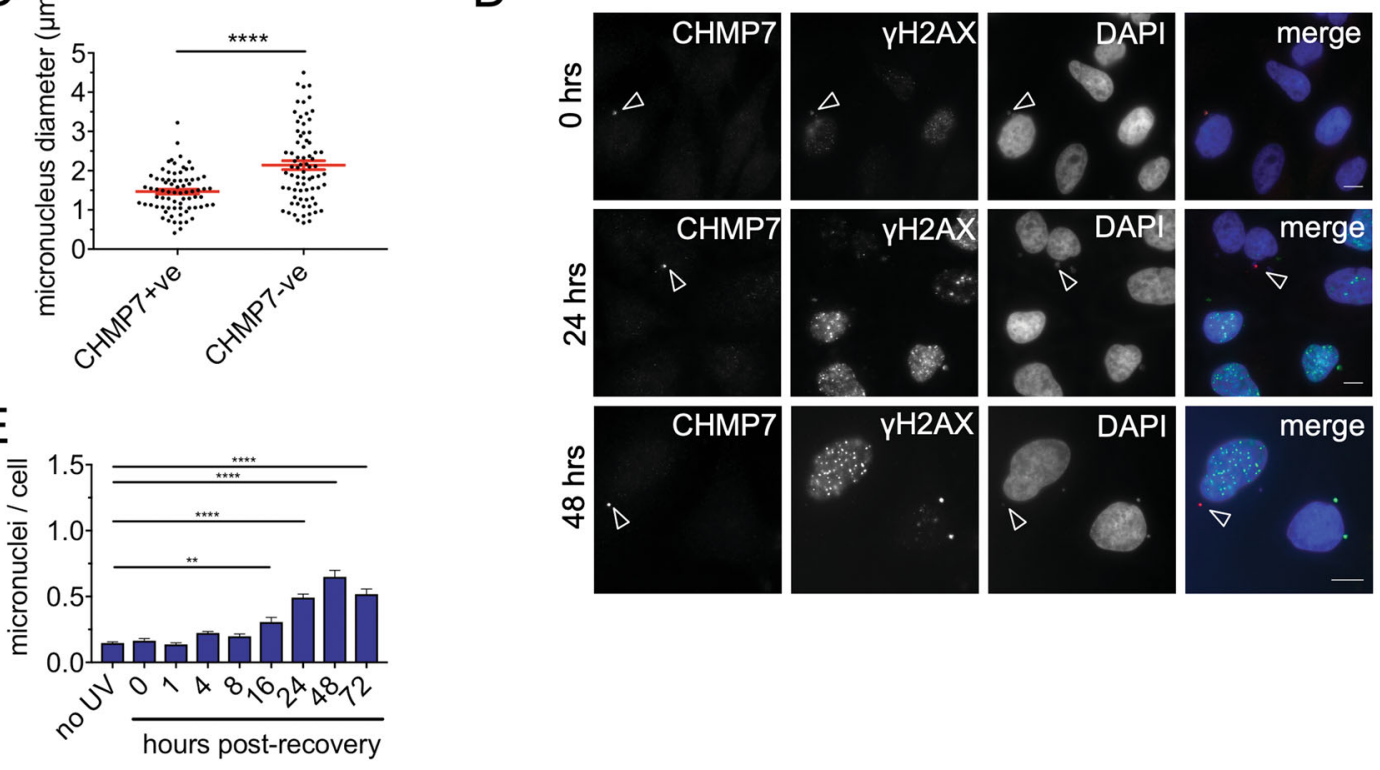

$\mathrm{F}$
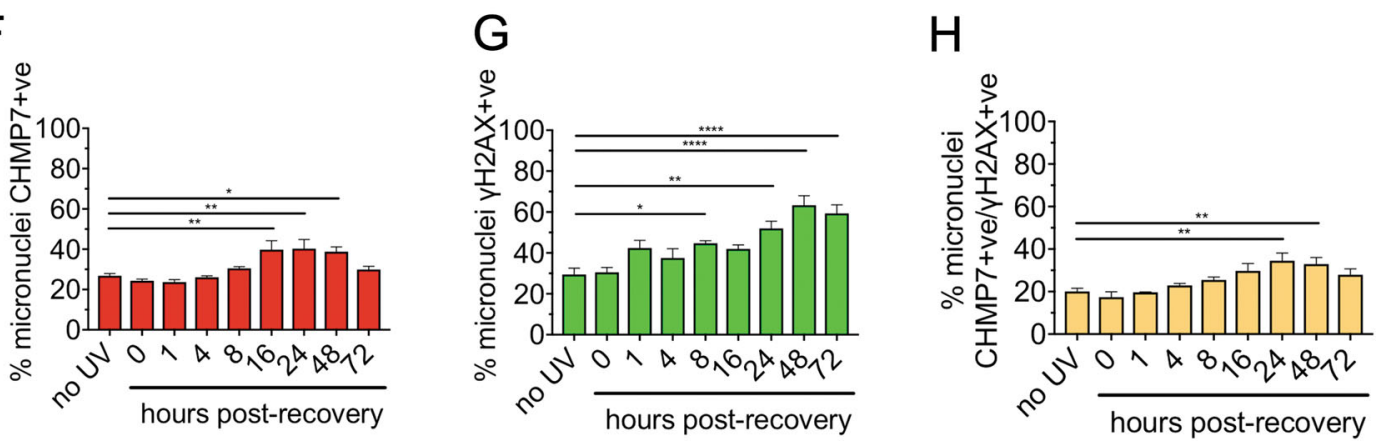

Fig. 6 ESCRT-III preferentially accumulates on acentric micronuclei. a HeLa cells transfected with either control or VPS4 sRNAi for $48 \mathrm{~h}$ hours and co-stained for CREST to detect centromeres, CHMP7, and DAPI. Examples of CHMP7 + ve micronuclei that are CREST + ve (arrowhead indicating weak CREST signal) or CREST-ve (arrowhead indicating the absence of CREST signal). Scale bars $10 \mu \mathrm{m}$. b Quantification of micronuclei in HeLa cells containing at least one definite CREST foci which resembles those found in the primary nucleus and CHMP7 (minimum 200 micronuclei scored per repeat). Results were analyzed using a Fishers exact test on pooled data. Individual biological repeats were also significant to Fishers exact $(p<0.05)$. $\mathbf{c}$ Control HeLa cells were stained for CHMP7 and DAPI. The diameter of micronuclei measured in ImageJ (75 CHMP7-positive and 75 CHMP7-negative micronuclei). HeLa cells were transfected with sRNAi for $48 \mathrm{~h}$ and stained to show CREST, CHMP7, and DAPI. $\mathbf{d}-\mathbf{h}$ HeLa cells were treated with a $10 \mathrm{~mJ} / \mathrm{cm}^{2}$ dose of UV-C irradiation and fixed at various timepoints following treatment. The cells were stained for $\gamma H 2 A X, C H M P 7$, and DAPI. d Accumulations of CHMP7 and $\mathrm{YH} 2 \mathrm{AX}$ within a single micronucleus in control cells and in cells 24 and $48 \mathrm{~h}$ post recovery from UV-C (scale bar $10 \mu \mathrm{m}$ ). The number of micronuclei per cell post UV-C treatment is shown in e. Micronuclei were scored for presence of $\mathbf{f} \mathrm{CHMP7}$ accumulation and $\mathbf{g}$ YH2AX foci, with micronuclei containing at least one focus being considered as positive (400 cells per treatment). The percentage of micronuclei positive for both $\mathrm{YH} 2 \mathrm{AX}$ and $\mathrm{CHMP7}$ are shown in $\mathbf{h}$. The percentage is calculated against the total number of micronuclei counted for each time point, varying between a minimum of 50 and a maximum of 285. Averages and SEM are shown $(N=3)$. Results were analyzed using a one-way ANOVA with Dunnett's post hoc test 

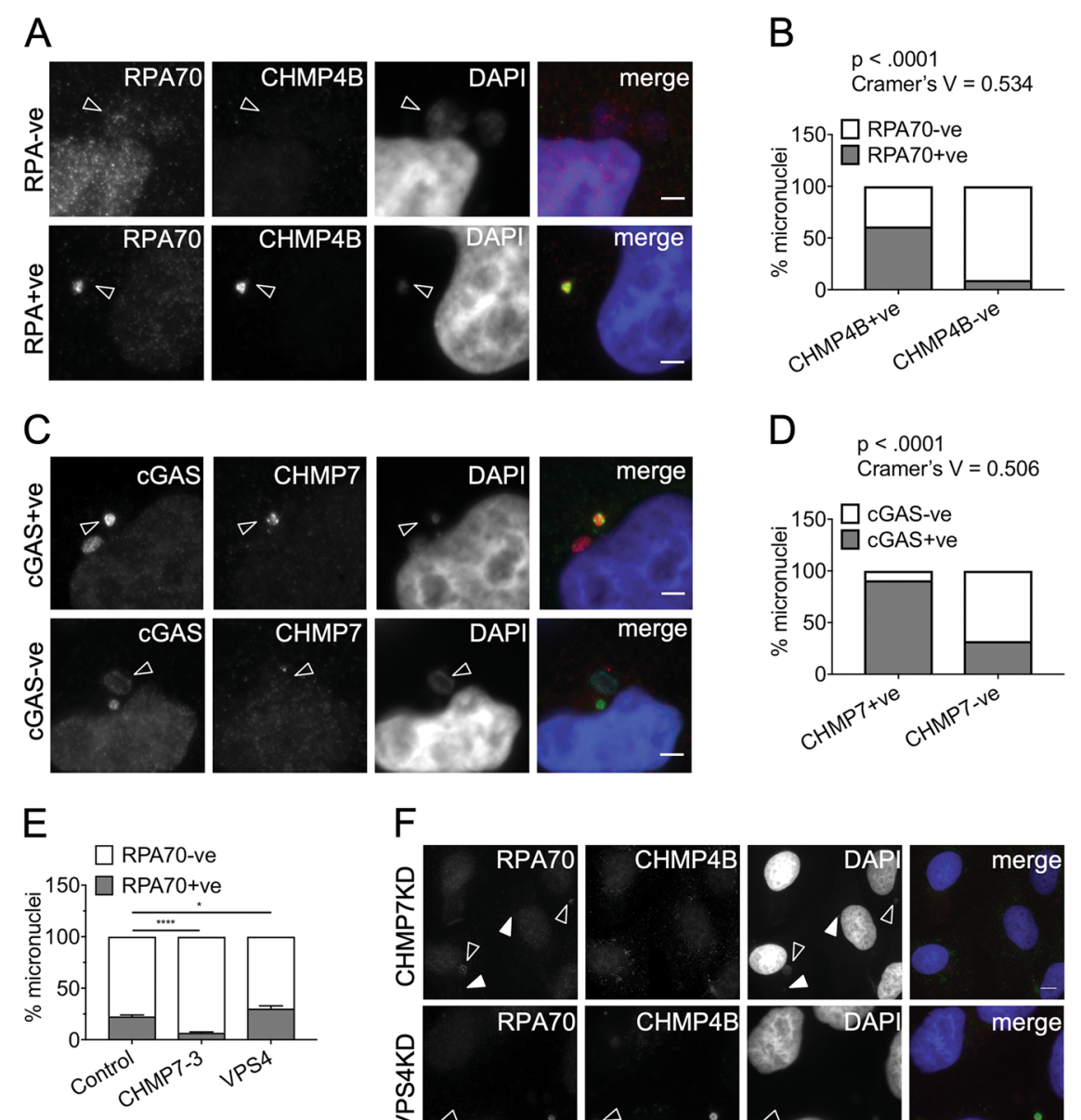

$\mathrm{F}$
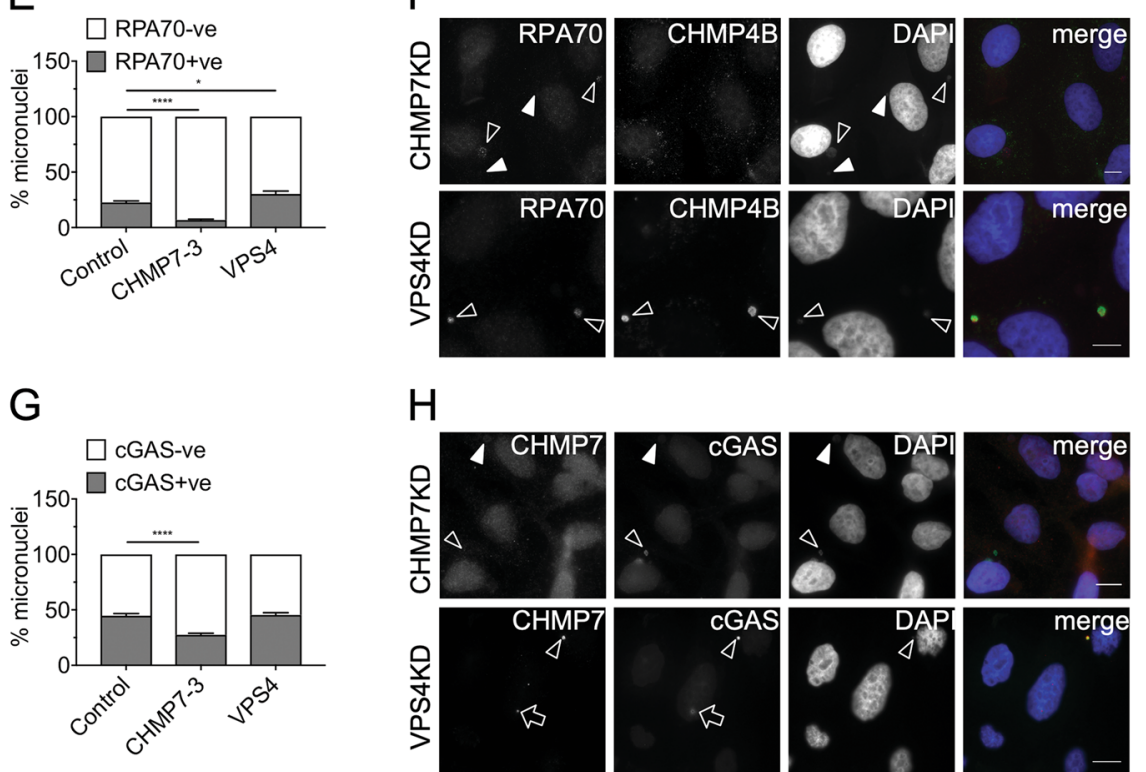

$\mathrm{H}$

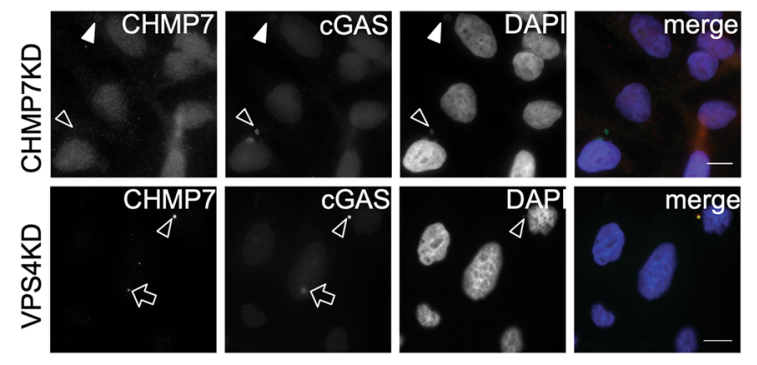

Fig. 7 (See legend on next page.)

f) or cGAS (Fig. 7g, h). In contrast, VPS4KD did not appreciably affect RPA or cGAS enrichment at micronuclei (Fig. 7e-h), despite its negative impact on micronuclear integrity (Supplemental Fig. 2B and Fig. 2H).

In summary, the co-enrichment of ESCRT-III and RPA/ cGAS at a subset of micronuclei and the absence of RPA/ cGAS enrichment upon CHMP7 depletion, point to a non-canonical (VPS4-independent) function for ESCRTIII in maintaining a pool of cytosolic DNA in cancer cells.

\section{Discussion}

We have addressed how the ESCRT-III membrane-repair complex supports genomic stability via its effects on aberrant structures associated with chromosomal instability and genotoxic events, namely micronuclei. Micronuclear collapse contributes to the accumulation of damaged DNA arising as a result of NE rupture, increasing the likelihood of failed DNA replication and persistent genomic instability. Therefore, the 
(see figure on previous page)

Fig. 7 CHMP7 is important for generating damaged DNA at micronuclei. a Control HeLa cells containing micronuclei positive or negative for RPA70 and CHMP4B. Top panel: the arrowheads show micronuclei negative for RPA70 and CHMP4B. Bottom panel: the arrowheads show micronuclei positive for RPA70 and CHMP4B. Scale bar $3 \mu \mathrm{m}$. b Micronuclei, in control HeLa cells, scored for the status of CHMP7 and for presence or absence of single-stranded DNA (RPA70) within the same micronucleus (minimum 115 micronuclei scored per repeat). Data analyzed using Fishers' exact test on pooled raw counts distributions $(N=3)$. c Control HeLa cells with micronuclei positive or negative for CGAS and CHMP7. Top panel: the arrowheads show micronuclei positive for CGAS (red) and CHMP7 (green). Bottom panel: the arrowheads show micronuclei negative for cGAS (green) and CHMP7 (red). Scale bar $3 \mu \mathrm{m}$. $\mathbf{d}$ Micronuclei, in control HeLa cells, scored for the status of CHMP7 and the presence or absence of cGAS within the same micronucleus (minimum 150 micronuclei scored per repeat). Data analyzed using Fishers' exact test on pooled raw counts distributions $(N=3)$. e Micronuclei from HeLa cells transfected for $48 \mathrm{~h}$ with the indicated siRNA and scored for enrichment in RPA70 signal (minimum 50 total micronuclei scored per treatment, per repeat). Results were analyzed using a one-way ANOVA with Dunnett's post hoc test. Averages and SEM shown ( $N=3$ ). $\mathbf{f}$ Top panel: HeLa cells depleted of CHMP7 for $48 \mathrm{~h}$ (arrowheads show RPA70 + ve micronuclei; filled arrowheads show RPA70-ve micronuclei). Bottom panel: HeLa cells depleted of VPS4 for $48 \mathrm{~h}$ (arrowheads show RPA70 + ve and CHMP4B + ve micronuclei). Scale bar $10 \mu \mathrm{m}$. g Micronuclei in HeLa cells transfected for $48 \mathrm{~h}$ with the indicated siRNA and scored for enrichment in CGAS signal (minimum 55 micronuclei scored per treatment, per repeat). Results analyzed using a one-way ANOVA with Dunnett's post hoc test. Averages and SEM shown $(N=3) \cdot \mathbf{h}$ Top panel: HeLa cells depleted of CHMP7 for $48 \mathrm{~h}$ (arrowheads show CGAS + ve micronuclei; filled arrowheads show CGAS-ve micronuclei). The CHMP7 channel is 3x over-exposed to highlight the absence of CHMP7 accumulations on the micronuclei. Bottom panel: HeLa cells depleted of VPS4 for $48 \mathrm{~h}$ (arrowhead shows a cGAS + ve micronucleus; arrow shows CGAS enrichment at a CHMP7 nuclear accumulation). Scale bar $10 \mu \mathrm{m}$. $\mathbf{f}$ and $\mathbf{h}$ the exposure time has been adjusted to compensate for varying intensity of the signals between CHMP7 and VPS4 knockdown experiments

existence of a mechanism for protecting the DNA within micronuclei is plausible, in order to avoid chromosome shattering and chromothripsis ${ }^{3,37}$.

Whereas the process of reformation of a micronuclear envelope is not well understood, almost $100 \%$ of spontaneously arising micronuclei display successful nucleocytoplasmic compartmentalization upon exit from mitosis ${ }^{2}$. These micronuclei must have a sealed NE, a process carried out at the primary nucleus by the CHMP7/ ESCRT-III/VPS4 system ${ }^{17}$. Indeed, ESCRT-III dynamics at lagging chromosomes during telophase appears nor$\mathrm{mal}^{38}$. We show here that loss of CHMP7 or VPS4 leads to increased occurrence of micronuclei with a ruptured and collapsed NE, supporting a role for ESCRT-III in sealing micronuclear envelopes (Supplemental Fig. 4A).

Importantly, however, our data also highlight a population of ESCRT-III that apparently is not subject to rapid, VPS4-dependent turnover, which accumulates within micronuclei (Supplemental Fig. 4B). Two questions arise: what mechanisms lead to the generation of this "persistent" pool of ESCRT-III, and does the pool have a selective role at micronuclei that is distinct from membrane repair?

To identify events that lead to ESCRT-III accumulations on micronuclei, we have comprehensively characterized the nature of these structures. Most have a broken lamina and are infiltrated by ER membranes, with a consequent loss of compartmentalization. These phenotypes are more rarely observed in micronuclei that lack CHMP7. Such defects might be owing to aberrant micronuclear NE assembly after mitosis ${ }^{5}$ and/or failure to repair a ruptured micronuclear NE during interphase ${ }^{2}$.

At the primary nucleus, the recruitment of ESCRT-III is so transient ${ }^{7,10}$ that depletion of VPS4 is necessary to slow down ESCRT-III turnover at the membrane (VPS4dependent pathway, Supplemental Fig. 4A). Our VPS4KD data imply the existence of a similar mechanism; hence, the accumulation of CHMP7 and CHMP4B at micronuclei observed in unperturbed cancer cells implies the normal balance between ESCRT-III assembly and disassembly is impaired and/or is not regulated by VPS4 (VPS4-independent pathway, Supplemental Fig. 4B).

ESCRT-III-enriched micronuclei are smaller than those lacking ESCRT-III and are predominantly acentric; they therefore most likely contain chromosome fragments, such as those generated by DNA damage in previous cell cycles $^{39}$. Hence, ESCRT-III accumulation might result from aberrant NE formation around chromosomal fragments, in which the NE membrane is subject to an unusually high degree of curvature and is prone to rupture ${ }^{40}$. One potential explanation for impaired ESCRT-III turnover at acentric micronuclei could be the lack of a regulatory activity that relies on signaling from the centromere.

Steric confinement provided by dense spindle microtubules at telophase causes uneven loading of NE proteins on lagging chromosomes, consequently generating micronuclei with a defective $\mathrm{NE}^{38}$. This phenomenon explains why such micronuclei may spontaneously rupture later in interphase; therefore, it is possible that ESCRT-III is recruited to micronuclei arising from this pathway.

Is there a role for aberrant ESCRT-III accumulations? ESCRT-III enrichment at micronuclei may cause further damage to DNA (Supplemental Fig. 4B), as suggested by the significant association between ESCRT-III and RPA, which marks the presence of single-stranded DNA ${ }^{41}$. Both RPA and cGAS enrichment in micronuclei are lost by depleting CHMP7 but not VPS4 (Fig. 7), arguing that the 
accumulation of ESCRT-III is required to generate higher levels of damaged DNA (Supplemental Fig. 4B).

The implications for aberrant NE remodeling by ESCRT-III at micronuclei may be far further reaching than simply explaining a genome predisposition to aneuploidy $^{19,42}$ or chromothripsis caused by loss of NE repair activity.

Ruptured micronuclei are key signals to activate immune pathways that can control tumor progression ${ }^{14,43}$ via the cGAS-STING (STimulator of Interferon Gene) pathway $^{14}$. cGAS recognition drives immune responses, senescence $^{44,45}$ and the elimination of cytosolic DNA by autophagy ${ }^{46}$. The ESCRT-III subunit CHMP4B was previously localized at micronuclei and a link with autophagic degradation of cytosolic DNA was suggested ${ }^{47}$.

CHMP7 depletion decreases the population of cGASenriched micronuclei. The observed reduction is similar in magnitude to the proportion of micronuclei with ESCRT-III accumulations in unperturbed cells $(\sim 20 \%)$. This suggests that ESCRT-III supports cGAS in ruptured micronuclei, perhaps by enhancing the production of DNA structures recognized by $\mathrm{CGAS}^{14,16}$. It is unlikely that CHMP7 depletion simply creates unstable micronuclei that lose cGAS, as VPS4 depletion does not reduce either the pool of RPA or cGAS-enriched micronuclei even though VPS4-depleted cells show loss of compartmentalization (Supplemental Fig. 2B and Fig. 2H).

In the context of cancer progression, short-lived cGAS signaling may be beneficial to induce antitumor responses; conversely, it may contribute to cancer progression if persistently sustained ${ }^{48}$. Higher CHMP7 and cGAS ${ }^{44}$ RNA expression correlate with higher frequency of patient survival in lung adenocarcinoma (Supplemental Fig. 4C, D), a tumor with high levels of chromosomal instability ${ }^{2,48}$. Moreover, CHMP7 has been identified as gene that negatively regulates cell proliferation (STOP gene), in a study showing how recurrent deletions occurring in cancers drive tumorigenesis ${ }^{49}$. The CHMP7 gene is located on the short arm of chromosome 8 (8p21.3). Chromosomal deletions on this region are common in carcinomas ${ }^{50-54}$ and loss of heterozygosity in $8 \mathrm{p}$ regulates tumor progression and drug resistance ${ }^{55}$. Therefore, it is intriguing to speculate that among the functions of ESCRT-III there is a role for aberrant membrane-remodeling activity that damages DNA, expose it to the cytosol, thus promoting the activation of proinflammatory pathways that modulate the tumor microenvironment.

\section{Methods}

\section{Cell lines, transfections, and sRNAi knockdowns}

HeLa, HeLa M, U2OS, and h-TERT cells were cultured in Dulbecco's Modified Eagle Medium (DMEM, Life Technologies) supplemented with $10 \%$ fetal bovine serum and $1 \%$ non-essential amino acids (Lonza). Cells were mycoplasma-free, with regular checks performed using the Mycoalert Mycoplasma Detection Kit (Lonza).

Depletion of target proteins was carried out using DHARMAFECT and ON-TARGETplus sRNAis per manufacturer's instructions (Thermo Scientific: Dharmacon Products, Lafayette, CO, USA) (see Supplementary Table 1 for sRNAi sequences). Cells were plated $24 \mathrm{~h}$ prior to knockdown, transfected once with sRNAi for 24-72 h at the appropriate concentration (usually $20 \mathrm{~nm}$ otherwise stated) dissolved in serum-free DMEM. The efficiency of sRNAi depletion was assessed by immunoblotting or quantitative PCR.

To visualize GFP-NLS in CHMP7 knockdown experiments, HeLa and h-TERT-immortalized Human Fibroblast cells were transfected with sRNAi CHMP7 $20 \mathrm{~nm}^{19}$ or control sRNAi (GLi2 $20 \mathrm{nM})^{56} 24 \mathrm{~h}$ after seeding, then transfected with $1 \mu \mathrm{g}$ pEGFP-NLS(SV40) (Kind gift from Dr. Patrizia Lavia) and fixed at $48 \mathrm{~h}$ post transfection.

\section{Quantitative PCR}

Quantitative PCR was used to assess VPS4A depletion in HeLa cells ${ }^{57}$. In brief, RNA was purified from cell pellets using the Absolutely RNA Microprep kit (Stratagene). First-strand cDNA was synthesized using random primers with the Multiple Temperature cDNA Synthesis Kit (Stratagene). Samples were stored at $-20^{\circ} \mathrm{C}$. RT-qPCR was performed using the SYBR-GREEN JumpStart Taq Ready Mix (Sigma). Conditions used were: initial denaturation at $94{ }^{\circ} \mathrm{C}$ for $2 \mathrm{~min}$, followed by 40 cycles of denaturation at $94^{\circ} \mathrm{C}$ for $15 \mathrm{~s}$, annealing at $53.7^{\circ} \mathrm{C}$ for $1 \mathrm{~min}, 72^{\circ} \mathrm{C}$ for $1 \mathrm{~min}$. Tata Binding Protein (TBP) was used as a loading control for the qPCR.

TBP amplification primers: forward, 5'-GCCCGAAAC GCCGAATATAATCCC-3'; reverse, 5'-GGACTGTTC TTCACTCTTGGCTCCTG-3'. VPS4A primers: forward, 5'-GACAACGTCAACCCTCCAG-3'; reverse, 5'-AGC GCCTCCTCGTAGTTCTT-3'.

Depletion of VPS4A was 98\% effective as judged by RT-PCR.

\section{Immunofluorescence microscopy}

Cells were fixed with $4 \%$ paraformaldehyde for $15 \mathrm{~min}$ at room temperature and permeabilized with $0.5 \%$ Triton X-100 for $20 \mathrm{~min}$. Primary (Supplementary Table 2) and secondary antibodies were incubated in $1 \%$ bovine serum albumin in phosphate-buffered saline containing $0.1 \%$ Triton X-100 in a humidified chamber at $4{ }^{\circ} \mathrm{C}$. Coverslips were mounted with VECTASHIELD (Vector Laboratories) or ProLong Gold (Invitrogen) mounting medium containing $1.5 \mu \mathrm{g} / \mathrm{ml}$ DAPI. Fluorescence was detected using a Nikon Eclipse TE200 microscope with $\times 60$ or $\times$ 100 objectives. Images were acquired using a Hamamatsu C4742-96-12G04 digital CCD camera and Volocity imaging software (Perkin Elmer), and analyzed using $\mathrm{Fij}^{58}$. 
An Applied Precision Deltavision deconvolution microscope with a UplanSApo $\times 100$ oil (NA 1.4) objective and a Photometrics CoolsnapHQ CCD camera was used to obtain 3D images. Z-slices were taken at $200 \mathrm{~nm}$ increments. Image acquisition was carried out using SoftWoRx v6 software, and deconvolution using Resolve 3D software at recommended settings. Images were analyzed using $\mathrm{Fi}_{\mathrm{ji}}{ }^{58}$.

\section{STED microscopy}

STED images of cells stained with To-Pro3 (Thermofisher) or Abberior STAR- (Abberior GmbH, Germany), Alexa Flour- or Oregon Green- (ThermoFisher Scientific, Australia) conjugated secondary antibodies diluted 1:300 were acquired with a Leica TCS SP8 microscope (Leica Microsystems $\mathrm{GmbH}$, Mannheim, Germany) using a Leica HCPL Apo $\times 100 / 1.40$ oil STED white objective, and equipped for 3D-gated- STED microscopy with a white light laser (tunable from $470 \mathrm{~nm}$ to $670 \mathrm{~nm}$ ) for excitation, $775 \mathrm{~nm}$ and $592 \mathrm{~nm}$ lasers for STED depletion and Hybrid Detectors. STED lasers were operated at $50 \%$ output power. Emitted fluorescence was filtered using notch filters $(775,592$, or $488 \mathrm{~nm})$. Images were acquired using sequential scanning with a line average of 2 , a frame accumulation of 3, and a scan speed of $600 \mathrm{~Hz}$. Images contained $1024 \times 1024$ pixels. Pixel size was $25 \mathrm{~nm}(\mathrm{X}, \mathrm{Y})$ and $67 \mathrm{~nm}(\mathrm{Z})$. Each $\mathrm{z}$ series (with sections at $67 \mathrm{~nm}$ intervals) was acquired using Leica Application Suite (LAS), then deconvolved using Huygens Professional software (Scientific Volume Imaging, The Netherlands). Images were analyzed using LAS and $\mathrm{Fij}^{5}{ }^{58}$.

\section{Micronuclei scoring}

Micronuclei from random fields of view in unsynchronized, untreated or sRNAi-treated cells were scored for enrichment in CHMP7 or CHMP4B and for a second marker. Only interphase, non-apoptotic cells were analyzed (cells containing $>3$ micronuclei were excluded to further minimize the possibility of including apoptotic cells). Micronuclei were defined by criteria based on Fenech et al..$^{59}$ : their diameter should not exceed $1 / 3$ that of the primary nucleus; they should be separated from the primary nucleus; they should have similar intensity of DAPI staining to the primary nucleus but occasionally may stain more intensively. Micronuclei were identified first by DAPI staining and were then scored for the presence or absence of CHMP7, appearing as an intense panmicronuclear signal or large foci. Other phenotypes were quantified based on visual assessment of various markers, with fluorescence intensity over the primary nucleus taken as a reference where appropriate.

\section{Statistical analysis of micronuclei categories}

Data were analyzed as in Hatch et $\mathrm{al}^{2}{ }^{2}$. In brief, the statistical difference between two or more categories of micronuclei was determined using Fisher's exact test (for a $2 \times 2$ table) or $\mathrm{x} 2$ analysis on the raw counts to obtain a $p$ value. The Null hypothesis assumed was that all phenotypes occurred with the same frequency. The Cramer V coefficient (or $\phi$ coefficient for a $2 \times 2$ table) was used to assess the strength of association between variables. The difference between independent replicates was determined by comparing $p$ values for each individual experiment. Raw counts were pooled and a final $p$ value calculated if the $p$ values of individual replicates resulted in statistical significance.

\section{Acknowledgements}

$B C$ and $\mathrm{HB}$ acknowledge the University of Sheffield for a PhD studentship to JW and the EPSRC for a studentship to AJC. BC acknowledges the EPSRC grant EP/M027821/1. PGW acknowledges the BBSRC grant BB/M000877/1. CR acknowledges the AIRC grant IG\#17739. The authors acknowledge the facilities and the scientific and technical assistance of the Australian Microscopy \& Microanalysis Research Facility at the Australian Centre for Microscopy \& Microanalysis at the University of Sydney and the Wolfson Light Microscopy Facility at the University of Sheffield.

\section{Author details}

'Department of Chemistry, Centre for Membrane Interactions and Dynamics (CMIAD), Krebs Institute, University of Sheffield, Brook Hill, Sheffield S3 7HF, United Kingdom. ${ }^{2}$ Academic Unit of Molecular Oncology, Sheffield Institute for Nucleic Acids (SInFoNiA), Department of Oncology and Metabolism, University of Sheffield, Beech Hill Road, Sheffield S10 2RX, United Kingdom. ${ }^{3}$ The

University of Sydney, Australian Centre for Microscopy and Microanalysis, Sydney, Australia. ${ }^{4}$ School of Biological Sciences, Faculty of Biology Medicine and Health, University of Manchester, Manchester Academic Health Science Centre, Manchester M13 9PT, United Kingdom. IBPMM-CNR c/o Universita' degli Studi di Roma Sapienza, Rome, Italy. ${ }^{6}$ Unit of Cellular Networks and Molecular Therapeutic Targets, IRCCS-Regina Elena National Cancer Institute, Rome, Italy

Conflict of interest

The authors declare that they have no conflict of interest.

\section{Publisher's note}

Springer Nature remains neutral with regard to jurisdictional claims in published maps and institutional affiliations.

Supplementary Information accompanies this paper at (https://doi.org/ 10.1038/s41389-019-0136-0).

Received: 21 November 2018 Revised: 28 February 2019 Accepted: 27 March 2019

Published online: 15 April 2019

\footnotetext{
References

1. Fenech, M. et al. Molecular mechanisms of micronucleus, nucleoplasmic bridge and nuclear bud formation in mammalian and human cells. Mutagenesis 26, 125-132 (2010).

2. Hatch, E. M., Fischer, A. H., Deerinck, T. J. \& Hetzer, M. W. Catastrophic nuclear envelope collapse in cancer cell micronuclei. Cell 154, 47-60 (2013).

3. Zhang, C.-Z. et al. Chromothripsis from DNA damage in micronuclei. Nature 522, 179-184 (2015).

4. Terradas, M., Martín, M. \& Genescà, A. Impaired nuclear functions in micronuclei results in genome instability and chromothripsis. Arch. Toxicol. 90, 2657-2667 (2016).

5. de Castro, I. J., Gil, R. S., Ligammari, L., Di Giacinto, M. L. \& Vagnarelli, P. CDK1 and PLK1 coordinate the disassembly and reassembly of the nuclear envelope in vertebrate mitosis. Oncotarget 9, 7763-7773 (2018).
} 
6. Terradas, M., Martín, M., Hernández, L., Tusell, L. \& Genescà, A. Nuclear envelope defects impede a proper response to micronuclear DNA lesions. Mutat Res. Fundam. Mol. Mech. Mutagen. 729, 35-40 (2012).

7. Raab, M. et al. ESCRT III repairs nuclear envelope ruptures during cell migration to limit DNA damage and cell death. Science 352, 359-362 (2016).

8. Robijns, J. et al. In silico synchronization reveals regulators of nuclear ruptures in lamin AVC deficient model cells. Sci. Rep. 6, 30325 (2016).

9. Denais, C. M. et al. Nuclear envelope rupture and repair during cancer cell migration. Science 352, 353-358 (2016).

10. Olmos, Y., Hodgson, L., Mantell, J., Verkade, P. \& Carlton, J. G. ESCRT-III controls nuclear envelope reformation. Nature 522, 236-239 (2015).

11. Olmos, Y., Perdrix-Rosell, A. \& Carlton, J. G. Membrane binding by CHMP7 coordinates ESCRT-III-dependent nuclear envelope reformation. Curr. Biol. 26 2635-2641 (2016)

12. Gu M., et al. LEM2 recruits CHMP7 for ESCRT-mediated nuclear envelope closure in fission yeast and human cells. Proc. Natl. Acad. Sci. USA 114, E2166-E2175 (2017).

13. Henne W. M., Stenmark H., Emr S. D. Molecular mechanisms of the membrane sculpting ESCRT pathway. Cold Spring Harbor Perspect. Biol. 5, pii: a016766 (2013).

14. Mackenzie, K. J. et al. cGAS surveillance of micronuclei links genome instability to innate immunity. Nature 548, 461-465 (2017).

15. Chen, Q., Sun, L. \& Chen, Z. J. Regulation and function of the CGAS-STING pathway of cytosolic DNA sensing. Nat. Immunol. 17, 1142-1149 (2016).

16. Civril, F. et al. Structural mechanism of cytosolic DNA sensing by cGAS. Nature 498, 332-337 (2013).

17. Vietri, M., Stenmark, H. \& Campsteijn, C. Closing a gap in the nuclear envelope. Curr. Opin. Cell Biol. 40, 90-97 (2016).

18. Vietri, M. et al. Spastin and ESCRT-III coordinate mitotic spindle disassembly and nuclear envelope sealing. Nature 522, 231-235 (2015).

19. Morita, E. et al. Human ESCRT-III and VPS4 proteins are required for centrosome and spindle maintenance. Proc. Natl. Acad. Sci. USA 107, 12889-12894 (2010).

20. De Vos, W. H. et al. Repetitive disruptions of the nuclear envelope invoke temporary loss of cellular compartmentalization in laminopathies. Hum. Mol. Genet. 20, 4175-4186 (2011).

21. Xu B., et al. Replication stress induces micronuclei comprising of aggregated DNA double-strand breaks. PLOS ONE 6, e18618-11 (2011).

22. Ward, I. M. \& Chen, J. Histone H2AX is phosphorylated in an ATR-dependent manner in response to replicational stress. J. Biol. Chem. 276, 47759-47762 (2001).

23. Wolf, C. et al. RPA and Rad51 constitute a cell intrinsic mechanism to protect the cytosol from self DNA. Nat. Commun. 7, 11752 (2016).

24. Shimizu N. Molecular mechanisms of the origin of micronuclei from extrachromosomal elements. Mutagenesis 26, 119-123 (2010).

25. Utani, K.-I., Okamoto, A. \& Shimizu, N. Generation of micronuclei during interphase by coupling between cytoplasmic membrane blebbing and nuclear budding. PLOS ONE 6, e27233-12 (2011).

26. Thoresen, S. B. et al. ANCHR mediates Aurora-B-dependent abscission checkpoint control through retention of VPS4. Nat. Cell Biol. 16, 550-560 (2014).

27. Agromayor M., Martin-Serrano J. Knowing when to cut and run: mechanisms that control cytokinetic abscission. Trends Cell Biol. 23(9):433-441 (2013).

28. Norppa, H. \& Falck, G. C.-M. What do human micronuclei contain? Mutagenesis 18, 221-233 (2003).

29. Bakhoum, S. F. et al. The mitotic origin of chromosomal instability. Curr. Biol. 24, R148-R149 (2014).

30. Shah P., Wolf K., Lammerding J. Bursting the bubble - nuclear envelope rupture as a path to genomic instability? Trends Cell Biol. 27, 546-555 (2017).

31. Gekara, N. O. DNA damage-induced immune response: micronuclei provide key platform. J. Cell. Biol. 216, 2999-3001 (2017).

32. Marti, T. M., Hefner, E., Feeney, L., Natale, V. \& Cleaver, J. E. H2AX phosphorylation within the G1 phase after UV irradiation depends on nucleotide excision repair and not DNA double-strand breaks. Proc. Natl. Acad. Sci. USA 103, 9891-9896 (2006)
33. Gabrielli, B. G., Clark, J. M., McCormack, A. K. \& Ellem, K. A. Ultraviolet lightinduced G2 phase cell cycle checkpoint blocks cdc25-dependent progression into mitosis. Oncogene 15, 749-758 (1997).

34. Harding, S. M. et al. Mitotic progression following DNA damage enables pattern recognition within micronuclei. Nature 548, 466-470 (2017).

35. Gelot, C., Magdalou, I. \& Lopez, B. Replication stress in mammalian cells and its consequences formitosis. Genes 6, 267-298 (2015).

36. Sabatinos, S. A., Ranatunga, N. S., Yuan, J.-P., Green, M. D. \& Forsburg, S. L. Replication stress in early $\mathbf{S}$ phase generates apparent micronuclei and chromosome rearrangement in fission yeast. Mol. Biol. Cell 26, 3439-3450 (2015).

37. Ly, $P$. et al. Selective $Y$ centromere inactivation triggers chromosome shattering in micronuclei and repair by non-homologous end joining. Nat. Cell Biol. 19, 68-75 (2017).

38. Liu, S. et al. Nuclear envelope assembly defects link mitotic errors to chromothripsis. Nature 561, 551-555 (2018).

39. Crasta, $\mathrm{K}$. et al. DNA breaks and chromosome pulverization from errors in mitosis. Nature 482, 53-58 (2012).

40. Xia, Y. et al. Nuclear rupture at sites of high curvature compromises retention of DNA repair factors. J. Cell Biol. 217, 3796-3808 (2018).

41. Haaf, T. et al. Sequestration of mammalian Rad51-recombination protein into micronuclei. J. Cell Biol. 144, 11-20 (1999).

42. Carlton, J. G., Caballe, A., Agromayor, M., Kloc, M. \& Martin-Serrano, J. ESCRT-II governs the Aurora B-mediated abscission checkpoint through CHMP4C. Science 336, 220-225 (2012).

43. Bakhoum, S. F. et al. Chromosomal instability drives metastasis through a cytosolic DNA response. Nature 553, 467-472 (2018).

44. Yang, H., Wang, H., Ren, J., Chen, Q. \& Chen, Z. J. cGAS is essential for cellular senescence. Proc. Natl. Acad. Sci. USA 114, E4612-E4620 (2017).

45. Glück S., et al. Innate immune sensing of cytosolic chromatin fragments through cGAS promotes senescence. Nat. Cell Biol. 19,1061-1070 (2017).

46. Bartsch, $\mathrm{K}$. et al. Absence of RNase $\mathrm{H} 2$ triggers generation of immunogenic micronuclei removed by autophagy. Hum. Mol. Genet. 26, 3960-3972 (2017).

47. Sagona, A. P., Nezis, I. P. \& Stenmark, H. Association of CHMP4B and autophagy with micronuclei: implications for cataract formation. Biomed. Res. Int. 2014 974393-10 (2014).

48. Bakhoum, S. F. \& Cantley, L. C. The multifaceted role of chromosomal instability in cancer and its microenvironment. Cell 174, 1347-1360 (2018).

49. Solimini, N. L. et al. Recurrent hemizygous deletions in cancers may optimize proliferative potential. Science 337, 104-109 (2012).

50. Emi, M. et al. Frequent loss of heterozygosity for loci on chromosome $8 p$ in hepatocellular carcinoma, colorectal cancer, and lung cancer. Cancer Res. 52 5368-5372 (1992)

51. Yaremko, M. L. et al. Loss of heterozygosity from the short arm of chromosome 8 is associated with invasive behavior in breast cancer. Genes Chromosomes Cancer 16, 189-195 (1996).

52. Kang, J. Genomic alterations on 8p21-p23 are the most frequent genetic events in stage I squamous cell carcinoma of the lung. Exp. Ther. Med. $\mathbf{9}$ 345-350 (2015).

53. Takle, L. A. \& Knowles, M. A. Deletion mapping implicates two tumor suppressor genes on chromosome $8 \mathrm{p}$ in the development of bladder cancer. Oncogene 12, 1083-1087 (1996).

54. Pineau, P. et al. Identification of three distinct regions of allelic deletions on the short arm of chromosome 8 in hepatocellular carcinoma. Oncogene $\mathbf{1 8}$ 3127-3134 (1999)

55. Cai, Y. et al. Loss of chromosome $8 p$ governs tumor progression and drug response by altering lipid metabolism. Cancer Cell. 29, 751-766 (2016).

56. Barbiero, I. et al. CDKL5 localizes at the centrosome and midbody and is required for faithful cell division. Sci. Rep. 7, 6228 (2017).

57. Stefani, F. et al. UBAP1 is a component of an endosome-specific ESCRT-1 complex that is essential for MVB sorting. Curr. Biol. 21, 1245-1250 (2011).

58. Schindelin, J. et al. Fiji: an open-source platform for biological-image analysis. Nat. Methods 9, 676-682 (2012).

59. Fenech, M. Cytokinesis-block micronucleus cytome assay. Nat. Protoc. 2 1084-1104 (2007) 